\title{
Chapter 46 \\ Chemical, Biological, Radiological \\ and Nuclear (CBRN) Casualty Management Principles
}

\author{
Steven A. Bland
}

\begin{abstract}
Chemical, biological, radiological or nuclear (CBRN) weapons have been used since antiquity. Examples of their recent use include war fighting, ethnic conflict, terrorism and assassination. In addition, CBRN incidents have also included accidental releases during peace time operations, and many of the principles for CBRN incident response can be applied to other hazardous material (HAZMAT) incidents. The impact of such weapons may have a range of implications for medical personnel both military and civilian.
\end{abstract}

Keywords Chemical weapons - Biological weapons - Radiological dispersal devices $\bullet$ Dirty bombs $\bullet$ Nuclear weapons $\bullet$ Terrorism $\bullet$ Weapons of mass destruction $\bullet \mathrm{WMD} \bullet \mathrm{CBRN} \cdot$ Hazardous materials $\bullet$ HAZMAT • Contamination • Contagious

\section{Objectives}

- To describe the characteristics and effects of CBRN agents

- To outline the principles of CBRN casualty management

- To outline the requirements for casualty hazard management including decontamination

This chapter is adapted from AMedP-6: NATO Management of CBRN Casualties Manual for a civilian readership

S.A. Bland, BSc, MBChB, MSc(MedTox), FCEM

Department of Medical, Defence CBRN Centre, Winterbourne Gunner,

Salisbury SP4 0ES, UK

e-mail: steven.bland239@mod.uk 


\section{Introduction}

Chemical, biological, radiological or nuclear (CBRN) weapons have been used since antiquity. Examples of their recent use include war fighting (World War One and the Iran-Iraq War), ethnic conflict (chemical weapon use against the Iraqi Kurds and in Syria), terrorism (release of sarin in the Tokyo underground, US anthrax letters) and assassination (ricin, polonium-210). In addition, CBRN incidents have also included accidental releases during peace time operations, and many of the principles for CBRN incident response can be applied to other hazardous material (HAZMAT) incidents. The impact of such weapons may have a range of implications for medical personnel both military and civilian. The health consequences related to a real or perceived CBRN hazard are:

- The initial (immediate, acute and delayed onset) health effects of CBRN agents

- The long-term (late onset or chronic) health effects of CBRN agents

- The secondary exposure risk to medical personnel due to either contamination or a contagious illness

- The requirement to carry out casualty hazard management that may include decontamination, isolation and quarantine

- The implementation of wider public health reporting and travel restrictions for transmissible biological agents

- The potential impact on the health of individuals due to use of CBRN personal protective equipment, including heat illness and psychological stress

- The potential impact on the healthcare system to implement pre-exposure or postexposure prophylaxis

- The management of any casualty, including trauma, in a CBRN environment

- The presence of combined (CBRN and conventional) injury

- The traumatic effects due to a nuclear detonation

Each type of medical mission will have its own risk assessment with a spectrum of hazards including CBRN weapons, environmental hazards ${ }^{1}$ and endemic disease as well as conventional injuries due to trauma; this all-hazards approach is reflected by chemical, biological, radiological, nuclear, environmental, endemic and traumatic hazards (CBRNEET). Some agents have no alternative uses other than as a weapon such as a nuclear weapon or sulphur mustard gas.

\section{Characteristics of CBRN Agents}

The medical effects of a CBRN agent depend on a number of characteristics that affect not only the medical presentation of casualties but also the delivery method,

\footnotetext{
${ }^{1}$ This includes toxic industrial hazards (TIH), also referred to as hazard materials (HAZMAT).
} 
type of response, physical protection and additional resources required such as decontamination or isolation. Important characteristics include agent class, physical properties and onset of effects (latency):

- Chemical agents: The main classes of chemical agents are:

- Nerve agents (organophosphorus compounds).

- Blistering agents (vesicants) agents.

- Cyanides (also known as blood agents).

- Pulmonary agents (choking or lung damaging agents).

- Incapacitants (mental and physical).

- Toxic industrial chemicals (TICs). While this class of agent is used, there is significant overlap with other classes of chemical agents especially cyanides and pulmonary agents.

- Riot-controlled agents (RCAs). These agents used by law enforcement agencies are not prohibited by international conventions but may still have harmful effects.

- Pharmaceuticals. This class includes illicit and commercial drugs usually at supra-therapeutic or toxic doses.

- Biological agents: The two classes of biological agent are:

- Live agents such as bacteria including rickettsia and chlamydia, viruses and fungi

- Toxins - chemical agents that are of biological origin and include those derived from bacteria, fungi, plants and animals (venom)

- Radiological material: This hazard can be classed by the type of ionising radiation present:

- Alpha - a relatively large subatomic particle (similar to a helium nucleus) with limited range in air (millimetres) but significant damaging effects

- Beta - a small subatomic particle similar to an electron with a range in air of centimetres

- Gamma/X-ray - high-energy photons with no mass but highly penetrating

- Neutrons - normally associated with nuclear material and the fission (nuclear) process which are highly penetrating and variable damaging effects

- Nuclear material: The term nuclear material is generally used to describe material involved in the nuclear power or weapon industry, or as having fissile properties, i.e. the potential for the nucleus to be split and therefore generate energy, fission products and further neutron emissions.

The physical properties of an agent may vary across a class of agent (e.g. nerve agents). These properties determine the optimal delivery system, route of exposure and continuing presence in the environment (persistency). The physical properties of an agent determine whether the agent is a: 
- Gas: A substance that exists in the air at ambient (room) temperature (nonpersistent hazard).

- Vapour: A substance that diffuses into the air (evaporates) but is normally a liquid at ambient (room) temperature (non-persistent hazard, but may condense into clothing and wound dressings).

- Liquid: Persistent hazard although the degree of persistency is dependent on volatility of the substance with water often considered a reference point (i.e. petrol $<$ water while water $<\mathrm{VX}$ ).

- Aerosols: Some solids and liquids can be aerosolised and therefore present a droplet or airborne hazard requiring respiratory protection. (These may remain a persistent hazard as particles settle on to surfaces with a risk of re-aerosolisation or evaporation if liquid.)

- Ionising radiation: Radioactive material may be in any of the physical forms just described, but irradiation itself does not present a contamination hazard.

\section{Methods of Delivery}

The characteristics of a CBRN agent such as physical properties and stability as well as vulnerable routes of absorption will determine the delivery method used. The device or munitions used may also cause additional medical effects such as trauma (injuries) or psychological stress. The two types of delivery are overt or covert. Overt releases are likely to follow a conventional major incident response, while a covert release may go unrecognised for a period of time. Subsequent response to a covert release may follow that for an outbreak using epidemiological methodology to establish cause and mitigation.

\section{Risk of Secondary Exposures (The Two Cs)}

For responders, CBRN incidents have significant risks due to the hazard. CBRN agents and other TIHs and endemic disease may be a continuing hazard to medical personnel. The main secondary hazards are:

- Contaminated casualties: Casualties coming into direct contact with a persistent agent will be contaminated (primary contamination). This may then be spread as secondary contamination to other personnel, equipment and infrastructure. Contamination may be classified as:

- External contamination: External contamination is the coating of an external surface such as the skin, hair or clothing of a casualty by a persistent agent. The agent may be liquid or dry particulate.

- Internal contamination: Internal contamination occurs by inhalation, ingestion or through the skin. Relatively small quantities of agent are internalised by the inoculation route and are unlikely to represent a secondary hazard. 
- Wound contamination: Wound decontamination is the introduction of a persistent agent into an area of traumatised tissue. A secondary hazard to responders including surgical teams may be present; however, the greater the potency of the agent, the less likely the casualty is to survive before surgery, and therefore the risk tends to be self-limiting. Devitalised (dead) tissue may also act as a buffer to limit systemic absorption of an agent.

- Contagious casualties: Casualties presenting with an infectious disease may pass the illness to others if the disease is transmissible. Mitigation is by isolation as decontamination will be ineffectual.

\section{Effects of Exposure (The Four Is)}

The type of CBRN agent will define the type of effects seen following an exposure, these are:

- Intoxication: This is due to a chemical (and toxin) exposure.

- Infection: This is due to a live biological agent exposure.

- Irradiation: This is due to ionising radiation exposure.

- Injuries: This is due to exposure to trauma or climatic stress (heat) either in isolation or as a combined injury (CBRN and trauma). The delivery method of the CBRN agent may cause trauma as well as physical degradation from individual protective equipment (IPE) use (heat injury).

\section{Onset of Effects (Latency)}

The onset of symptoms is important in the recognition and establishment of causation of a CBRN or TIH exposure. However, delay in these symptoms due to a latency period will confound any epidemiological investigation. For live biological agents, the latency period is called the incubation period. In some cases, such as certain live biological agents and acute radiation syndrome, there is a significant period of non-specific symptoms and signs (e.g. fever and muscle aches) that precede a more recognisable syndrome (or toxidrome); this is called a prodromal stage. A toxidrome is a pattern of symptoms and signs (syndrome) due to exposure to a toxic substance. Notable toxidromes are those for nerve agent intoxication and opioid overdose. Figure 46.1 provides a summary of the latency and incubation periods for certain CBRN agents.

\section{Medical Effects of CBRN Agents}

The medical effects of CBRN agents depend on a number of factors including route of exposure, type of agent and severity of effects. 


\begin{tabular}{|c|c|c|}
\hline \multirow[t]{2}{*}{ Chemical } & Chlorine & Intoxication \\
\hline & \multicolumn{2}{|l|}{ Phosgene } \\
\hline \multicolumn{2}{|c|}{ Nerve agents } & \\
\hline Cyanide & Sulphur mustard & \\
\hline
\end{tabular}

\begin{tabular}{|c|c|c|}
\hline Biological (toxins) & Ricin & Intoxication \\
\hline & tulinum toxin & \\
\hline
\end{tabular}

\begin{tabular}{|c|c|}
\hline Biological (live) & Infection \\
\hline Plaque & Tularemia \\
\hline Smallpox & \\
\hline Anthrax & Melidoidosis \\
\hline
\end{tabular}

\begin{tabular}{|c|c|c|}
\hline Radiological/nuclear & & Irradiation \\
\hline High Doses $>2$ Sv ARS prodrome & Acute radiation syndrome & $\uparrow$ Cancer risk \\
\hline Low doses & & 个 Cancer risk \\
\hline
\end{tabular}

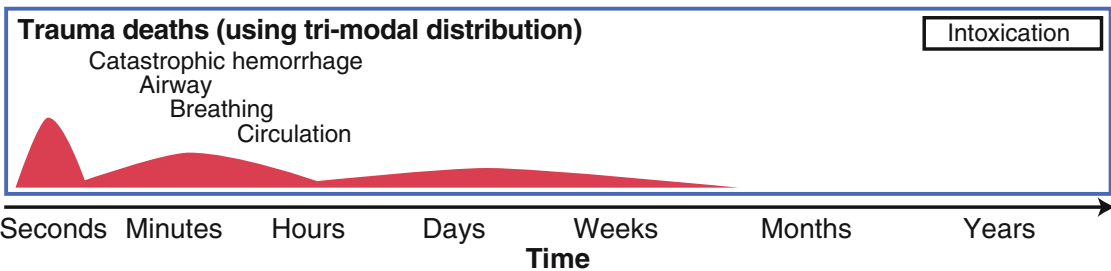

Fig. 46.1 Onset of effects of some CBRN agents (Courtesy of S.A. Bland)

\section{Routes of Exposure}

The routes of exposure (sometimes referred to as routes of absorption) are important both for predicting and modelling the medical effects of agents and the provision of protective equipment specific to that route. The routes of exposure are:

- Inhalation: This route may present the greatest risk of multiple casualties due to an airborne hazard such as a gas, vapour, aerosol, droplets (in close proximity) or smoke. Protection is by mask, respirator or self-contained breathing apparatus (SCBA).

- Ingestion: This route is less likely to cause mass fatalities due to the dilution of any chemical agent or destruction by the cooking process or digestion. It may, however, be a significant route for the introduction of live biological agents with high infectivity. This route may be used for the targeting of individuals or small numbers. Protection is provided by water and food security and the restriction of eating and drinking during and post-attack.

- Skin (percutaneous): There are three methods for CBRN agents to pass through the skin: 
- Intact skin (transcutaneous): Although skin is protective to many CBRN agents, some agents may directly damage the skin (vesicants) or have chemical properties (fat soluble) to allow penetration.

- Inoculation: This is the intentional break in the skin in order to introduce a CBRN agent causing minimal trauma.

- Wounds: This is the gross loss of skin integrity allowing for the opportunistic entry of CBRN agents into the body.

- Mucous membranes: Although skin provides significant protection to biological agents, mucous membranes, which form the lining of internal cavities such as the mouth and rest of the gut, are more permeable and may allow an effective dose to be absorbed. Moisture on mucous membranes may also be reactive with some chemical agents such as chlorine causing immediate effects.

- Eyes: While eyes are not a good route for absorption, there are significant local effects on the eyes and conjunctiva. An example is the pinpoint pupils (miosis) associated with nerve agent vapour.

\section{Severity of Effects}

The severity of effects is defined as:

- Lethal: These agents have a significant risk of causing death even at low level exposures and/or without treatment. The level of lethality can be defined as:

- Lethal dose $50\left(L D_{50}\right)$ : This is the dose required, usually expressed as weight per $\mathrm{kg}$ or dose, to kill $50 \%$ of the exposed population; the smaller the value, the greater the lethality. The LD50 is usually specific to a route of exposure, e.g. skin or ingestion. LD50 may also be expressed over a time period such as $\mathrm{LD}_{50 / 30}$ (30 days), and this is often used for irradiation.

- Lethal concentration time $50\left(L C t_{50}\right)$ : For the inhalation route, $\mathrm{LCt}_{50}$ is used as it reflects the inverse relationship between concentration and the duration of exposure to cause the same effect (death) in $50 \%$ of the exposed population.

- Case fatality rate (CFR): This is the number of deaths compared to the total number of cases, e.g. estimated pandemic influenza (3-4\%) and inhalational anthrax (95-100\%).

- Damaging: These effects are those that may not be associated with a high lethality but require significant medical resources, e.g. sulphur mustard.

- Incapacitating: These agents cause a reversible mental or physical disability and inability to function. Secondary effects may also occur and include trauma due to increased risk taking behaviour or heat illness due to the physiological effects of the agent. Examples include BZ (mental incapacitant) and adamsite (vomiting agent/physical incapacitant).

- Iatrogenic: These effects are due to the adverse drug effects of MedCMs. In all cases, the decision to implement a MedCM at a command or clinical level will 
be based on a risk-benefit assessment; for example, a MedCM such as atropine with significant side effects may be used to mitigate a lethal CBRN agent.

\section{Psychological Effects}

CBRN agents may cause a variety of psychological effects. Some effects may be appropriate to the hazard (i.e. acute stress reaction) and may even enhance the response to an incident. Other effects will cause mental incapacitation with symptoms ranging from anxiety (acute stress disorder) to acute psychosis or delirium. Symptoms can occur at any time and include post-traumatic stress disorder (PTSD). Psychological effects due to CBRN agents may be direct, indirect (reactive and degradation) and psychogenic.

\section{Summary of Chemical Agent (and Associated Chemicals), Effects and Management}

See Tables 46.1, 46.2, 46.3, 46.4, 46.5 and 46.6 for summaries of nerve agents (tabun, sarin, soman, VX), cyanides, sulphur mustard, pulmonary (lung damaging) agents (chlorine, phosgene), anticholinergics (atropine, BZ) and methaemoglobin formers (nitrites, other TICs)

Table 46.1 Nerve agents (tabun, sarin, soman, VX)

\begin{tabular}{|c|c|c|c|}
\hline \multirow{4}{*}{\multicolumn{2}{|c|}{$\begin{array}{l}\text { Mechanism: inhibition of the enzyme acetylcholines- } \\
\text { terase that breaks down the nerve transmitter } \\
\text { acetylcholine. This results in overstimulation of the } \\
\text { parasympathetic system, motor neurons (leading to } \\
\text { paralysis) and central nervous system }\end{array}$}} & \multicolumn{2}{|l|}{ QUICK LOOK } \\
\hline & & Consciousness & Seizures \\
\hline & & Resp rate & $\uparrow \uparrow$ \\
\hline & & Eyes & Pinpoint \\
\hline Mild & Miosis, eye pain, red eyes & Secretions & +++ \\
\hline Moderate & $\begin{array}{l}\text { Secretions, wheezing, nausea, } \\
\text { vomiting, diarrhoea, difficulty in } \\
\text { breathing }\end{array}$ & Skin & Sweaty \\
\hline Severe & $\begin{array}{l}\text { Muscle weakness, respiratory } \\
\text { fatigue, respiratory arrest, } \\
\text { seizures, death }\end{array}$ & \multicolumn{2}{|c|}{$\begin{array}{l}\text { Other factors: for skin exposure - } \\
\text { local fasciculation }\end{array}$} \\
\hline Antidotes & \multicolumn{3}{|c|}{$\begin{array}{l}\text { Anticholinergics (atropine), oximes (e.g. pralidoxime, obidoxime, HI-6), } \\
\text { benzodiazepines (diazepam) }\end{array}$} \\
\hline Other agents & \multicolumn{3}{|c|}{$\begin{array}{l}\text { Organophosphorus pesticides, main route of exposure is ingestion, but } \\
\text { possible airborne dissemination with varying effect. Treat as noted } \\
\text { although pharmacokinetics may vary due to dose and route of exposure }\end{array}$} \\
\hline
\end{tabular}


Table 46.2 Cyanides

\begin{tabular}{|c|c|c|c|}
\hline \multirow{4}{*}{\multicolumn{2}{|c|}{$\begin{array}{l}\text { Mechanism: cyanide inhibits the mito- } \\
\text { chondrial enzymes in cells. This stops } \\
\text { cells using oxygen and metabolising } \\
\text { glucose completely (aerobic respira- } \\
\text { tion). This leads rapidly to a metabolic } \\
\text { (lactic) acidosis } \\
\end{array}$}} & \multicolumn{2}{|l|}{ QUICK LOOK } \\
\hline & & \multirow{2}{*}{\begin{tabular}{|l} 
Consciousness \\
Resp rate
\end{tabular}} & \multirow{2}{*}{ Seizures/unconscious } \\
\hline & & & \\
\hline & & & N/dilated \\
\hline Mild & $\begin{array}{l}\text { Nausea, dizziness, } \\
\text { agitation }\end{array}$ & Secretions & Normal \\
\hline Moderate & $\begin{array}{l}\text { Hyperventilation, } \\
\text { confusion }\end{array}$ & Skin & Pink or cyan \\
\hline Severe & $\begin{array}{l}\text { Loss of consciousness, } \\
\text { seizures, coma, } \\
\text { respiratory arrest, death }\end{array}$ & \multicolumn{2}{|c|}{ Other factors: VERY RAPID ONSET (secs) } \\
\hline Antidotes & \multicolumn{3}{|c|}{$\begin{array}{l}\text { Oxygen, dicobalt edetate, hydroxocobalamin (vit B12), sodium/amyl nitrite and } \\
\text { sodium thiosulphate }\end{array}$} \\
\hline \multirow[t]{2}{*}{ Other agents } & \multicolumn{3}{|c|}{$\begin{array}{l}\text { Hydrogen sulphide (HS): bad egg gas that also causes aerobic respiration } \\
\text { failure }\end{array}$} \\
\hline & \multicolumn{3}{|c|}{$\begin{array}{l}\text { Phosphine gas: produced by contact of aluminium phosphide and moisture, } \\
\text { used widely as a rodenticide (not to be mistaken with phosgene gas) and } \\
\text { similar effects to cyanide }\end{array}$} \\
\hline
\end{tabular}

Table 46.3 Sulphur mustard (blistering agent)

\begin{tabular}{|c|c|c|c|}
\hline \multirow{2}{*}{\multicolumn{2}{|c|}{$\begin{array}{l}\text { Mechanism: Damage to DNA resulting in cell death } \\
\text { of exposure tissue including skin and airway } \\
\text { mucosa }\end{array}$}} & \multicolumn{2}{|l|}{ QUICK LOOK } \\
\hline & & \multirow{3}{*}{\begin{tabular}{|l|} 
Consciousness \\
Resp rate \\
\end{tabular}} & \multirow{3}{*}{\begin{tabular}{|l|}
- \\
$\uparrow$ \\
Normal
\end{tabular}} \\
\hline & & & \\
\hline & & & \\
\hline Mild & Erythema (red skin), eye pain & Secretions & Normal $/ \uparrow$ \\
\hline Moderate & $\begin{array}{l}\text { Skin blistering (small area), airway } \\
\text { irritation }\end{array}$ & Skin & Red/blisters \\
\hline Severe & \begin{tabular}{|l|}
$\begin{array}{l}\text { Airway burns/obstruction, large } \\
\text { area blisters }\end{array}$ \\
\end{tabular} & \multirow{2}{*}{\multicolumn{2}{|c|}{$\begin{array}{l}\text { Other factors: note mustard has delayed } \\
\text { onset }-12-24 \mathrm{~h}\end{array}$}} \\
\hline Delayed & $\begin{array}{l}\text { Immunosuppression, acute } \\
\text { respiratory distress syndrome }\end{array}$ & & \\
\hline Antidotes & \multicolumn{3}{|l|}{ None } \\
\hline Other agents & \multicolumn{3}{|c|}{$\begin{array}{l}\text { Lewisite: based upon arsenic and causes immediate pain and silver-grey } \\
\text { discolouration. May be lethal. (Antidote: dimercaprol), other chelating } \\
\text { agents }\end{array}$} \\
\hline & \multicolumn{3}{|c|}{$\begin{array}{l}\text { Hydrofluoric acid (HF): causes chemical burn with immediate symptoms and } \\
\text { hypokalaemia. (Antidote: calcium gluconate and calcium chloride) }\end{array}$} \\
\hline
\end{tabular}


Table 46.4 Pulmonary (lung damaging) agents (chlorine, phosgene)

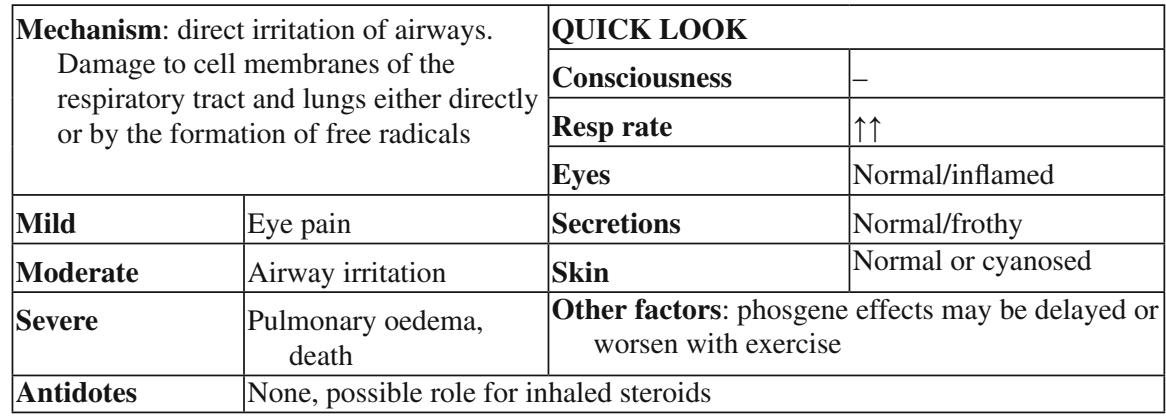

Table 46.5 Anticholinergics (atropine, BZ)

\begin{tabular}{|l|l|l|l|}
\hline \multicolumn{2}{|l|}{$\begin{array}{l}\text { Mechanism: blocking anti-muscarinic receptors } \\
\text { reducing parasympathetic tone and CNS effects }\end{array}$} & QUICK LOOK \\
\cline { 3 - 4 } & Consciousness & Confused \\
\cline { 3 - 4 } & Resp rate & Normal \\
\cline { 3 - 4 } Mild & Eyes & Dilated \\
\hline Moderate & Dry mouth, altered (blurred) vision & Secretions & Dry \\
\hline Severe & $\begin{array}{l}\text { Severe disorientation and risk of } \\
\text { heat illness } \\
\text { "Mad as a hatter, blind as a bat, dry } \\
\text { as a bone, red as a beet, hot as } \\
\text { hell" }\end{array}$ & $\begin{array}{l}\text { Other factors: confusion and possible } \\
\text { hallucinations }\end{array}$ \\
\hline Antidotes & Supportive treatment, reassurance. AVOID physical restrain. Physostigmine \\
\hline
\end{tabular}

Table 46.6 Methaemoglobin formers (nitrites, other TICs)

\begin{tabular}{|c|c|c|c|}
\hline \multirow{3}{*}{\multicolumn{2}{|c|}{$\begin{array}{l}\text { Mechanism: turns } \mathrm{Fe}^{2+} \mathrm{Hb} \text { into } \mathrm{Fe}^{3+} \text { Met-Hb. } \\
\text { This prevents the red blood cells carrying } \\
\text { oxygen from the lungs to the tissues }\end{array}$}} & \multicolumn{2}{|l|}{ QUICK LOOK } \\
\hline & & \multirow{2}{*}{\begin{tabular}{|l|} 
Consciousness \\
Resp rate \\
\end{tabular}} & \multirow{2}{*}{\begin{tabular}{|l} 
Agitated \\
$\uparrow / \uparrow \uparrow$
\end{tabular}} \\
\hline & & & \\
\hline & & & \\
\hline Mild & No obvious effect & Secretions & Normal \\
\hline Moderate & Cyanosis and shortness of breath & Skin & Blue \\
\hline Severe & $\begin{array}{c}\text { Severe cyanosis and shortness of } \\
\text { breath, confusion, death }\end{array}$ & \multicolumn{2}{|c|}{$\begin{array}{l}\text { Other factors: cyanosis not improved with } \\
\mathrm{O}_{2} \text {. Chocolate-coloured blood }\end{array}$} \\
\hline Antidotes & \multicolumn{3}{|c|}{ Methylene blue } \\
\hline
\end{tabular}

\section{Principles of CBRN Medical Incident Management}

A CBRN incident has significant security and intelligence implications; however, with regard to incident management, the presence of a CBRN agent or TIM is simply another hazard that requires mitigation. A conventional incident response incorporates safety considerations and a standardised incident report that includes hazard assessment. Where there is a combined hazard (explosive and CBRN), the 

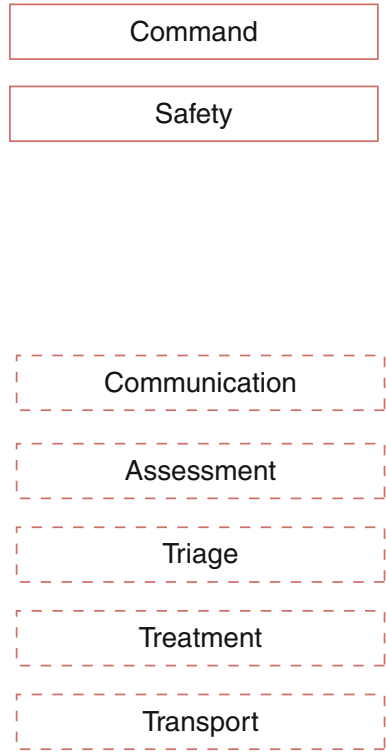
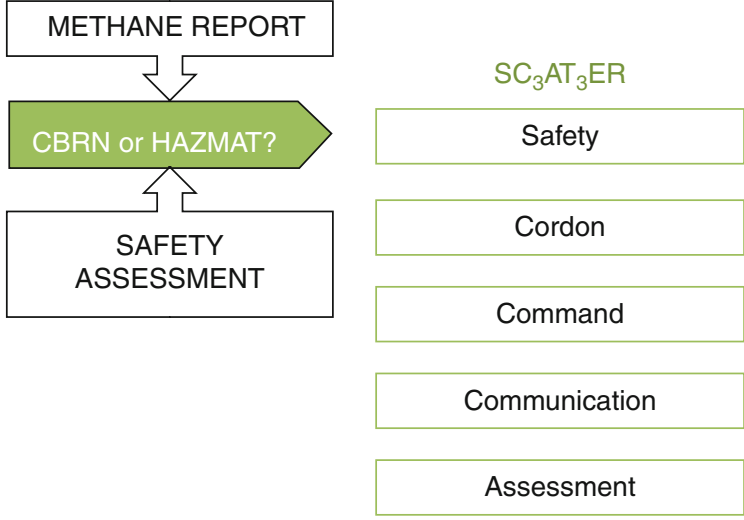

\begin{tabular}{|c|}
\hline Triage \\
\hline \\
\hline Treatment \\
\hline Transport \\
\hline Exploitation \\
\hline
\end{tabular}

Recovery

Fig. 46.2 Summary of CBRN medical incident management

presence of the CBRN agent may not be initially recognised, and an all-hazards approach is important. For covert releases, including outbreaks, an approach using epidemiological concepts is more likely. For an incident identified as a CBRN incident, the response can be optimised by modifying the conventional incident response. The principles of a $\mathrm{CBRN}$ medical incident response $\left(\mathrm{SC}_{3} \mathrm{AT}_{3} \mathrm{ER}\right)$ (Fig. 46.2) are:

- Safety: This includes evacuation of non-essential persons from the area, personal protective equipment (PPE) and hazard management. For all incidents, safety is of paramount importance, and safety assessments should be repeated.

- Cordons: This includes establishing hot, warm and clean zones as well as casualty decontamination areas (Fig. 46.3). For a significant biological incident with risk of person to person spread, cordons may support public health control measures with restriction of movement (ROM). ROM may include sovereign boundaries in the case of Public Health Emergencies of International Concern (PHEIC) as defined by the International Health Regulations (2005). The zones are defined as:

- The hot zone is a non-permissive area where there is a direct hazard from the environment (firearm, explosive hazard or CBRN). This zone is sometimes referred to as the exclusion zone especially where PPE does not mitigate the hazard such as in the case of explosives or high dose radiation. 


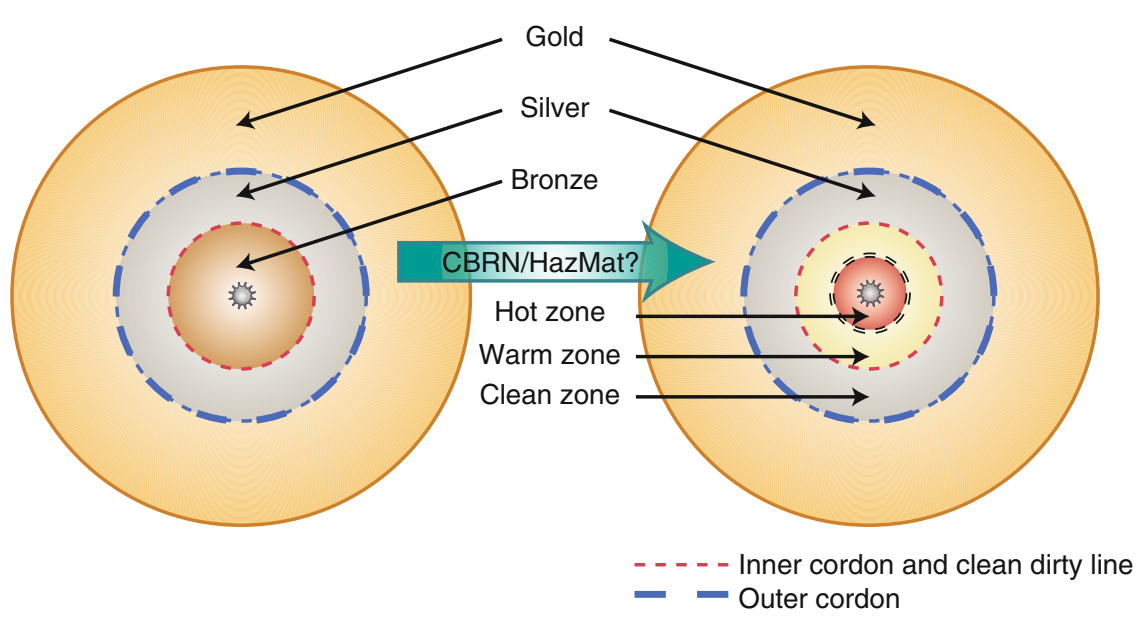

Fig. 46.3 CBRN and conventional zones. The conventional bronze zone in the diagram is contained by a inner cordon. It will be split into CBRN hot and warm (decontamination) zones contained by an inner cordon which is also the clean dirty line (Reproduced by permission S.A. Bland)

- The warm zone is a semi-permissive (buffer) area setup usually due to a continuing contamination hazard from casualties or equipment coming out of the hot zone. This zone is also referred to as the decontamination zone and demarcated by a clean dirty line $(\mathrm{CDL})$.

- Command and communications: Each zone and area will require a vertical and horizontal command structure to allow for interoperability between specialist units and the chain of command. The chain of communication should mirror the chain of command and include updates following further safety assessments and on identification or exclusion of any hazard.

- Assessment: Concurrent environmental and casualty assessment is important and both have advantages and disadvantages with detection and diagnosis, respectively. Any report of a CBRN agent must include the source of the information (environmental sampling, diagnosis) and the confidence of the assessment (suspected, probable, confirmed).

- Triage: See discussion to come.

- Treatment: Treatments of individual casualties and agents vary between nations and are subject to national legislation. However, examples of significant chemical agents and suggested initial management are listed at the end of this chapter.

- Transport: The presence of a CBRN agent and risk of secondary contamination or infection has significant implications for casualty transport especially by air. Some methods may also be limited by aviation and international regulation.

- Exploitation and recovery: As well as the treatment of CBRN casualties, another implication of a CBRN event is the potential loss or compromise of a medical facility such as a hospital. The priority after casualty management has been completed is to recover back to an original state while also exploiting the scene for forensic evidence. 


\section{Principles of CBRN Casualty Management}

The principles of CBRN casualty management are:

- Recognition (detection \& diagnosis)

- Safety (personal and collective)

- Self-aid/first aid

- Triage

- Casualty assessment - 'quick look'

- Life-saving interventions

- Casualty hazard management

- Supportive treatment

- Definitive treatment

- Rehabilitation

The provision of CBRN casualty care starts from point of exposure (PoE) and may continue to rehabilitation. The ability to provide care from PoE to hospital depends on the limitations imposed by the operational environment due to the presence of a primary or secondary hazard. Care within contaminated areas is limited to first aid and Emergency Medical Treatment (EMT).

The main zones (Fig. 46.3) using an all-hazards approach are described as:

- Hot zone (non-permissive) management: Casualty care in a non-permissive environment is limited by the direct risk to the responder and the protective measures or actions required (individual protective equipment, return of fire). This level of care is sometimes described as "Care under Fire (CUF)" with hazards including CBRN (primary exposure), an explosive device, effective fire or environmental. Management is limited to:

- Self- and first aid

- Triage

- Casualty assessment ('quick look' include a trauma primary survey)

- Life-saving interventions (T1 casualties) for CBRN and trauma

- Evacuation to the Casualty Collection Point (CCP)

- Warm zone (semi-permissive): Casualty care is limited due to the residual hazard from casualties or equipment (contamination, contagious disease) and personal protective equipment. This level of care is sometimes called "tactical field (medical) care" and may take place at the CCP before decontamination or during the decontamination process. It is limited to:

- Triage

- Casualty assessment ('quick look' including Trauma Primary Survey)

- Life-saving interventions (T1 casualties)

- Casualty hazard management, e.g. decontamination

- Wound management 
- Evacuation to the Casualty Clearing Station (CCS) or a medical treatment facility (MTF).

- For specific agents and depending on evacuation timelines, MedCM may be given to prevent the deterioration of $\mathrm{T} 2 / 3$ casualties.

- Clean zone (permissive): Casualty care beyond the CDL allows optimal access to the patient, although treatment may still be limited by PPE due to wound contamination or contagious disease. Advanced medical care at this level is divided into:

- Supportive care: During CBRN casualty management, the causative agent may not be known. Treatment may still be effective by adequate supportive treatment focused on managing observed effects such hypoxia and hypotension. Even when the agent is known, there may be no definitive treatment, and treatment remains supportive throughout the continuum of care including critical care with advanced respiratory and circulatory support.

- Definitive care: Definitive treatment is the final level of care provided to return the patient to the highest degree of mental and physical capability possible. It includes further antidote treatment, replacement therapy, surgery and burns management. After definitive treatment, the casualty may undergo rehabilitation before being returned to duty. For some casualties such as irradiation and burns, definitive care may only be available at Role 4, in an allied nation or international network of medical facilities.

\section{CBRN Recognition}

Indications of a CBRN or other environmental hazards include:

- Any symptoms involving incident response personnel or Recce teams

- Multiple casualties with similar non-traumatic symptoms and signs

- Unusual taste, smell or mist

- Unexplained dead animals

- Unexplained symptoms including altered vision, eye pain, headache, chest tightness, excessive secretions and nonthermal burns

- Any unusual or unexplained symptoms, signs, morbidity or mortality

Different detection elements will also contribute to the recognition of a CBRN event, and some of these are provided by medical personnel. There is a cyclical relationship, and it is referred to as the cycle of recognition and is summarised in Fig. 46.4.

\section{CBRN Emergency Medical Treatment (EMT)}

The provision of prehospital medical support to CBRN casualties including trauma in a CBRN environment is described as EMT. EMT consists of: 


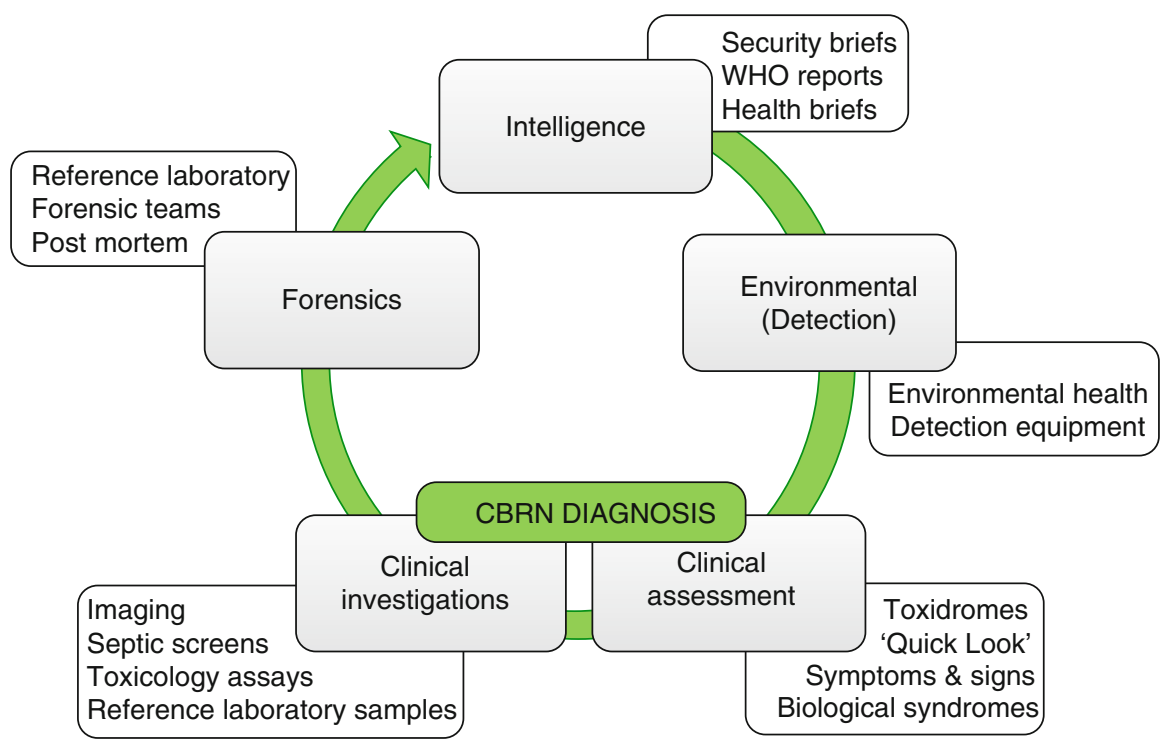

Fig. 46.4 Cycle of CBRN recognition

- Triage

- Casualty assessment ('quick look')

- Life-saving interventions (LSIs)

- Casualty hazard management

Medical treatment is provided for T1 casualties in the hot and warm zone, at the $\mathrm{CCP}$, during decontamination, at the CCS and during casualty evacuation, as required. The treatment is likely to be concurrent with decontamination possibly provided by non-medical personnel at the CCP or in the stretcher decontamination lane (see section "Casualty hazard management").

\section{Triage}

For any major incident including CBRN as demand increases, there is a requirement to allocate the finite resources available on a priority basis. This ensures that the casualties with the greatest need are treated first. The allocation of resources is based on the sorting (triage) of casualties. To support this, casualties exposed to a hazardous substance can be labelled as mild (T3), moderate (T2) and severe (T1), based on the known effects of specific agents. Triage categories are used for:

- The prioritisation for treatment

- The prioritisation for decontamination

- The prioritisation for casualty evacuation (transport) 
Table 46.7 CBRN triage categories and features

\begin{tabular}{|c|c|c|c|c|c|}
\hline \multirow[b]{2}{*}{ Category } & & \multicolumn{3}{|l|}{ Severity } & \multirow[b]{2}{*}{ Implications } \\
\hline & & Toxicity ${ }^{\mathrm{a}}$ & Sepsis & Radiation & \\
\hline $\mathrm{T} 1$ & Immediate & Severe & $\begin{array}{c}\text { Severe sepsis } \\
\text { or shock }\end{array}$ & $\begin{array}{l}\text { Other criteria } \\
\pm>2 \mathrm{~Gy}^{\mathrm{b}}\end{array}$ & $\begin{array}{l}\text { Life-saving interventions } \\
\text { Stretcher decontamination }\end{array}$ \\
\hline $\mathrm{T} 2$ & Urgent & Moderate & Sepsis & $>2 \mathrm{~Gy}$ & $\begin{array}{l}\text { Stretcher decontamination, } \\
\text { including incapacitated } \\
\text { casualties }\end{array}$ \\
\hline T3 & Delayed & Mild & Pyrexia & $<2 \mathrm{~Gy}$ & Walking decontamination \\
\hline T4 & Expectant & - & - & $>8 \mathrm{~Gy}$ & $\begin{array}{l}\text { "T1(hold)" or palliative } \\
\text { care. Colour used varies } \\
\text { with nations }\end{array}$ \\
\hline DEAD & Dead & - & - & - & - \\
\hline
\end{tabular}

aThe severity of the toxicity of a chemical exposure may be set by generic features (abnormal physiological parameters, convulsions, profound cyanosis and respiratory distress) and specific triage criteria specific to a chemical agent such as the presence of secretions for nerve agent exposure (see section "Summary of chemical agents")

${ }^{b}$ Dose estimation is based upon a combination of physical dosimetry, clinical signs, haematological parameters and chromosomal aberrations

CBRN incidents use the same categories as conventional incidents to ensure consistency and ensure trauma, CBRN and combined casualties have the same casualty flow priorities. Table 46.7 shows the triage categories and features of each. The method of triage system used will depend on the CBRN incident zone, accessibility to the casualty and safety factors. A standardised approach with consistent triage categories is recommended from PoE to transport to a specialist unit, with likely choke points at decontamination, admission to intensive care and surgery. The levels of triage are:

- Life sign assessment

- Triage sieve (algorithm based) (see also Chap. 35)

- Triage for decontamination (algorithm based plus contamination burden)

- Triage sort (scoring system using physiological parameters)

- Triage for surgery (extended triage sort, clinical investigations and surgical opinion)

- Triage for intensive care (extended triage sort and end-organ function)

- Triage for specialist management, including burns care and advanced radiation therapy

In the context of this chapter, the first 3 are of particular importance.

\section{Casualty Assessment: 'quick look'}

The Quick Look is a rapid focused initial assessment of a casualty for signs of trauma and intoxication. In the context of trauma, the assessment is for any catastrophic haemorrhage or respiratory distress due to an airway or breathing problem. Recognition of chemical casualties may be based upon the clinical signs of certain 
Table 46.8 Abbreviated Quick Look

\begin{tabular}{llllll}
\hline & Nerve & Cyanide & Opioid & Atropine & Sepsis \\
\hline Conscious & Fitting & $\downarrow \downarrow /$ Fitting & $\downarrow$ & $\uparrow$ & $\mathrm{N} / \downarrow$ \\
Resps & $\downarrow \downarrow / \downarrow$ & $\uparrow \uparrow \uparrow / \downarrow$ & $\downarrow \downarrow$ & $\mathrm{N} / \uparrow$ & $\uparrow \uparrow$ \\
Eyes & Pinpoint & N/Large & Pinpoint & Large & $\mathrm{N}$ \\
Secretions & $\uparrow \uparrow$ & $\mathrm{N}$ & $\mathrm{N}$ & Dry & $\mathrm{N} /$ sputum \\
Skin & Sweaty & Pink/blue & N/blue & Flushed & Sweating/pale \\
Other & D\&V & Sudden onset & & & High temperature \\
& bradycardia & & & & \\
\hline
\end{tabular}

$N$ normal, $D \& V$ diarrhea and vomiting

chemical agents using conscious level, respiratory pattern, eyes, secretions and skin (CRESS). Table 46.8 provides an abbreviated Quick Look for important CBRNrelated agents and drugs. While the incident management for biological incidents is likely to be over a slower time frame, casualty assessment of life-threatening infections and sepsis is vital for life-saving treatment. Septic shock is a life-threatening condition, and fluid resuscitation should be started within $10 \mathrm{~min}$ of diagnosis in the warm zone/CCP.

\section{Life-Saving Interventions}

LSIs are actions that can be performed by any appropriately trained person to reverse life-threatening conditions or prevent deterioration due to a CBRN agent and/or trauma. A generic approach can be applied to both CBRN and trauma using the following priorities for treatment:

- $<\mathbf{C}>$ atastrophic haemorrhage.

- Airway management and antidotes

- Breathing

- Circulation

- Decontamination and disability

- Evacuation to more permissive environment Immediate life-threatening conditions include:

- Catastrophic haemorrhage $(<\mathrm{C}>)$

- Airway obstruction (A)

- Breathing difficulty (B) including:

- Cyanosis (B)

- Respiratory distress (B)

- Tension pneumothorax (B)

- Sucking chest wound (B)

- Circulatory failure (C) due to:

- Shock due to loss of fluid or sepsis (C) 
- Bradycardia due to nerve agent $(\mathrm{C})$

- Disability (d) including:

- Unconscious (d)

- Convulsions (a and d)

- Other signs of severe chemical intoxication, where an effective MedCM exists and a delay in administration may cause death (a)

LSIs should only be performed on the most severe casualties (T1) with immediate life-threatening conditions and include:

- Removal from the hazard (self-extraction or rescue)

- Application of tourniquet(s) $(<\mathrm{C}>)$

- Application of pressure dressing and haemostatic agents $(<\mathrm{C}>)$

- Basic airway management including suction (A)

- Early MedCM/antidote administration including anticonvulsant (a)

- Ventilation (as resources allow) (B)

- Administration of oxygen (B)

- Management of tension pneumothorax, such as needle decompression (B) ${ }^{2}$

- Management of sucking chest wound, such as application of dressing with valve (B)

- Fluid resuscitation (trauma, sepsis or combined injuries) (C)

- Management of severe sepsis and septic shock (fluids, oxygen and antibiotics) (C)

Hot zone casualty care is limited to the management of T1 casualties with catastrophic haemorrhage, airway problems, antidote administration and management of sucking chest wound and/or tension pneumothorax followed by evacuation to the warm zone and the forward Casualty Collection Point (fwd CCP). These measures have the acronym $<\mathrm{C}>\mathrm{AaBE}$. The fwd $\mathrm{CCP}$ is a key ad hoc point in the casualty evacuation chain where medical management rather than first aid can take place as the casualty starts to be decontaminated. In many circumstances, evacuation "scoop and run" to a more permissible environment may be a more effective intervention than "stay and play" in the hot zone.

\section{Casualty Hazards Management}

Casualty hazard management is the decision-making process for the handling of casualties with a secondary exposure risk due to either contamination or a contagious illness (Fig. 46.5). LSIs take priority over casualty hazard management although the removal of chemical contamination may reduce further exposure to the casualty and the responder and are life-saving also. The components of casualty hazard management are:

\footnotetext{
${ }^{2}$ In the CBRN environment, a tension pneumothorax should be suspected in any casualty with a penetrating chest injury (or suspected blast injury) and respiratory distress or circulatory shock.
} 
Fig. 46.5 Casualty hazard management

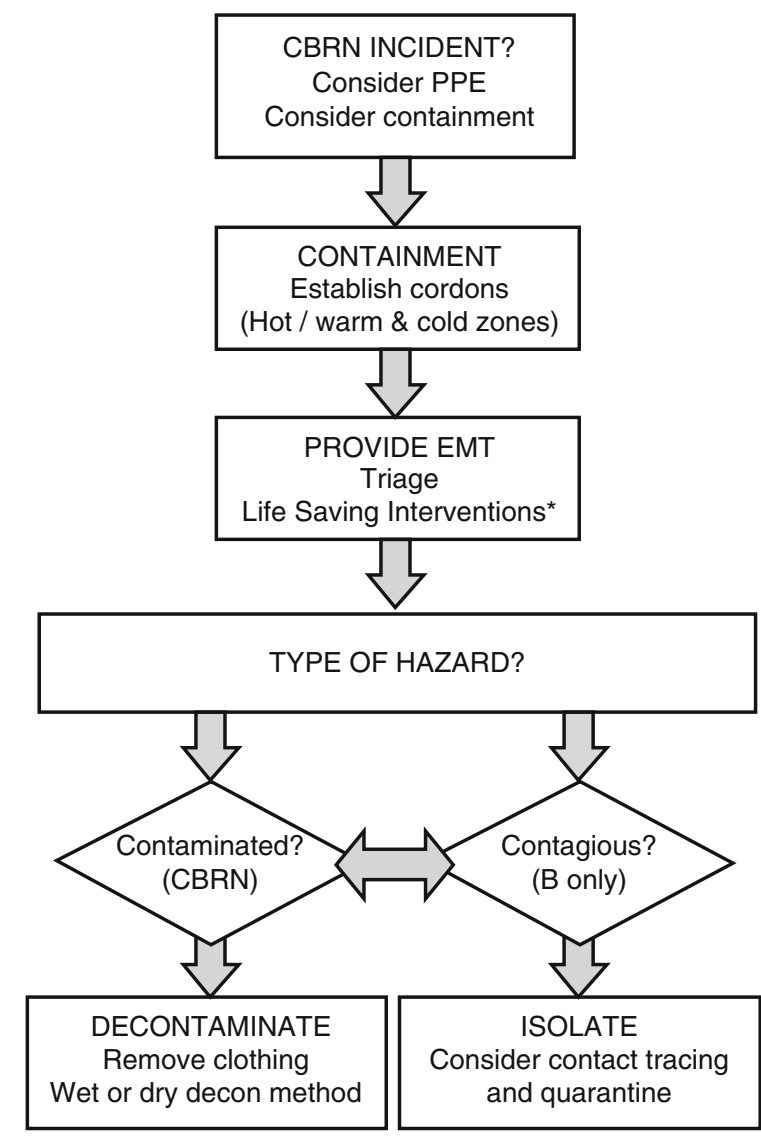

* includes remove from hazardous area

- Containment

- Decontamination

- Isolation

- Quarantine

- Public health control measures

\section{Containment}

Containment is the immediate on-scene action to limit further spread. It should not, however, prevent the clearance of persons away from any immediate threat such as an IED or armed enemy. Containing the scene and exposed persons allows the Incident Commander to assess the risks of secondary spread and the requirement for decontamination. 


\section{Decontamination}

Decontamination can be divided into external, internal (decorporation) and wound decontamination. External methods include:

- Physical removal: This is the mechanical removal of persistent chemical, biological and radiological contamination. The most common mechanical methods used alone or in combination are:

- Removal of clothing.

- Irrigation with copious amounts of water.

- Dry adsorbents. Adsorbents draw in liquid contaminants, and examples include fullers' earth and baking powder. It should be noted that the agent is not destroyed and an off-gassing hazard remains after dry decontamination.

- Chemical destruction: This method involves the deactivation of an agent by altering its structure. This can be achieved by chemical reactions such hydrolysis, oxidation and active decontaminants.

\section{Wound Decontamination}

The management of wounds and the use of tourniquets and dressings mean that the potential for external contamination exists, and all dressings and tourniquets must either be replaced or covered in a clear well-marked protective dressing or wrap. Copious water or weak hypochlorite solution is recommended for initial wound irrigation. Skin decontaminants such as Fuller's Earth and some active decontaminants should be avoided in wounds due to complications and potential delayed wound healing. Surgical management of any traumatic injury should include a wide debridement (removal of dead tissue), whether CBRN or conventional. There is a theoretical risk that contaminated wounds (munitions fragment or impregnated clothing) may pose a threat to a surgical team. For light contamination, this is very unlikely. Standard surgical procedures including the use of aseptic technique and surgical instruments, such as forceps, will minimise any risk. Research has demonstrated that a single pair of latex surgical gloves may not provide adequate protection from some chemical agents where there is direct contact, i.e. wound probing, and therefore double nitrile gloves, which have greater chemical resistance, are the minimum requirement.

\section{Isolation and Quarantine}

In the context of CBRN casualty hazard management, isolation refers to the separation of casualties with an illness due to a suspected transmissible (contagious) biological agent. The principles of isolation include physical protection and hazard management. Isolation will generally use negative pressure, and barrier nursing 
methods will be used and supported by the infection prevention and control nurses. Where there is more than one casualty with the same illness, a cohort facility or ward should be considered to reduce staffing and logistical demands. In the context of CBRN casualty hazard management and endemic disease, quarantine refers to the separation and observation over a period of time (usually the latency or incubation period) of a well person who may have been exposed to a suspected hazard or have an epidemiological link to a probable or confirmed case. Depending on the type of agent, severity, transmissibility and numbers exposed, the quarantined person may be under medical care, a responsible or regulatory organisation or agency. The latter may be the case for international travel as was seen during the SARS epidemic.

\section{Casualty Decontamination Area}

The requirement and level of casualty decontamination depends on the hazard present (hot/warm zone), persistency of the agent, protective equipment the casualty was wearing and decontamination method available (dry versus wet decontamination).

Elements of a CDA (Fig. 46.6) include:

- Commander (Officer or NCO), may be secondary role

- Casualty Collection Point

- Triage

- Ambulatory channel(s)

- Stretcher channel(s) with provision for EMT

- Body handling area

- Expectant (T4) area, as required

- Equipment decontamination

- Logistic support, as required

- Miscellaneous, including scribes (clerks) and runners

The clean dirty line (CDL) is the cordon at which point full decontamination (casualty, personnel and equipment) has taken place. The CDL is effectively the edge of the hazardous zone, sometimes referred to as the bronze zone and as such is also the inner cordon (Fig. 46.3).

For casualty management, this may be the first point that casualty documentation may have been generated, and a formal handover of care should take place. The handover should include as much information as possible and the minimum (AT-MIST) is:

- Age (if known)

- Time of exposure/injury and duration

- Mechanism of exposure, i.e. type of incident

- Injuries, intoxication, infection and irradiation suspected

- Symptoms and signs

- Treatment including antidotes and antibiotics given 


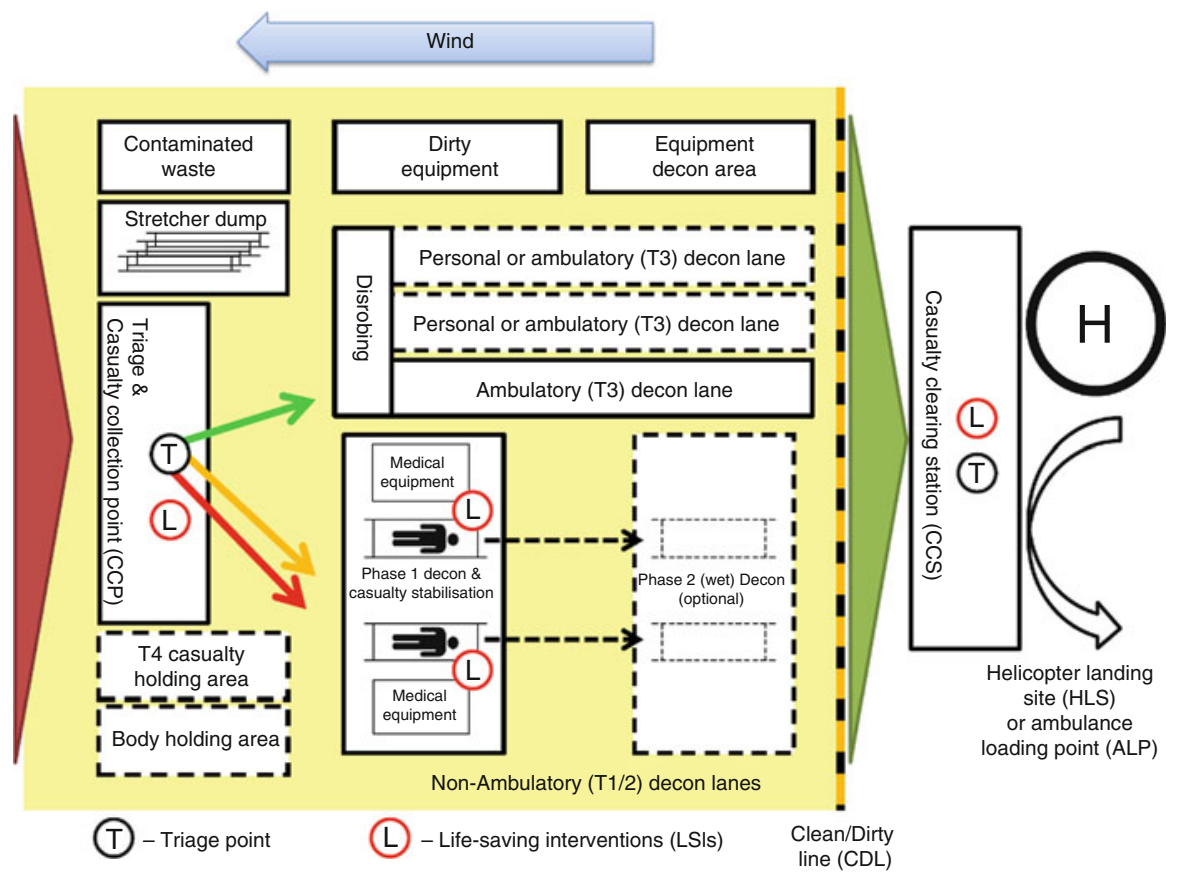

Fig. 46.6 Casualty decontamination area (with courtesy of S.A. Bland)

Although crossing the CDL implies decontamination has been completed, this should also be formally declared and any additional hazards such as wound or dressing contamination (or contagious in some cases) must be highlighted and recorded.

\section{Post-CBRN Event Actions}

\section{Occupational Medical Advice}

Following a CBRN incident, medical personnel may need to provide advice on measures including:

- Postexposure prophylaxis of responders that may have been exposed during the incident such as vaccination, antibiotics or anti-radiation countermeasures

- PPE to limit exposure of recovery workers

- Monitoring of the health aspects of working in a hazardous environment and PPE including heat illness 
- Advice and monitoring hazard management measures such as limiting working times

- Pre-exposure prophylaxis of recovery workers to CBRN and other occupational exposures including hepatitis A and B

- Recording any MedCM use and potential exposures

\section{Health Surveillance}

There may be a requirement to start appropriate health surveillance by using health registries. Registries may be based upon a cohort of the population that share the same potential or confirmed exposure to a hazard substance or environment or the same symptoms, syndromes or specific illness. Members of the register are either casualties that have been entered in to the registry by the receiving hospital or other potentially exposed persons that volunteer to be added to the register. The benefit of this is to:

- Identify any delayed or long-term health effects including mental health.

- Communicate rapidly between exposed persons and health organisations.

- If there are no observed health effects, reassure the exposed population.

- Mitigate any delay health effects as they are identified in other members of the cohort.

- Comply with potential health and occupational regulations.

\section{Aftercare of Responders}

As soon as possible after being stood down, all responders should be checked to ensure there are no injuries or acute effects following any potential hazardous exposure. In addition, all responders' details should be logged and kept in case of health follow-up and any likely investigations into the incident and its response. Where there is a known hazard, responders should be given advice on health feature to look out for and where necessary primary or preventive health care informed. Following any significant incident and especially where there may have been multiple casualties and/or CBRN, stress is likely to have a number of effects on individual, and this is to be expected. The management of psychological stress should be managed by the team in a supportive way that initially is independent of any medical interventions. Many post incident stress management systems use a series of escalating interventions, and these may be of benefit:

- Voluntary team debriefings

- Unit commander risk assessment of individual 
- Unit level counselling

- Referral for formal counselling

- Formal medical referral and interventions

\section{Further Reading}

CBRN incidents: clinical management and health protection handbook. Health Protection Agency. NATO Management of CBRN Casualties Handbook (AMedP-7.1). http://www.phe.gov.uk. 